\title{
Acceptability of revalidation to GDPs
}

Revalidation of general dental practitioners in Scotland: The results of a pilot study Part 2 - feasibility of operation Y. G. Maidment, J. S. Rennie and M. Thomas Br Dent J 2006; 200: 456-459

\section{Aim}

To investigate the acceptablility of a pilot scheme of revalidation to general dental practitioners.

\section{Method}

Ten general dental practitioners completed portfolios of evidence of being up to date and fit to practise. This portfolio was assessed by a panel of three experts, using an assessment tool developed specifically for that purpose. An action research methodology was used to evaluate participants' perceptions, consisting of a focus group and semistructured interviews. The views of the assessors on the portfolio and its assessment were collected using a questionnaire.

Results

The views of the participants on revalidation, the pilot scheme portfolio and its use, who should assess it and how its use could be supported were collected. Also areas of difficulty in using the portfolio were identified, along with suggestions for improving it and alternative ways of evidencing competence. Assessors noted that the quality of evidence was adequate, but also made suggestions for improvement of the portfolio.

\section{Conclusions}

The pilot scheme appears to have been acceptable to the dentists in this scheme, given some caveats. The assessors felt that appraisal would significantly enhance any substantive scheme.

\section{IN BRIEF}

- Revalidation is more robust than recertification.

- The process of revalidation will give a structure to continuing professional development.

- Appraisal or mentoring of revalidees by peers was seen as essential to the acceptability and feasibility of the process.

\section{COMMENT}

Revalidation is generally agreed to be a process by which a professional demonstrates that they are fit to continue practice in their field of work. The General Medical Council (GMC) launched a revalidation process for general medical practitioners based mainly on appraisal by local peers, and the General Dental Council (GDC) Revalidation Working Group has made considerable progress towards a model of revalidation for general dental practitioners.

These two papers present a pilot study of a possible model for the revalidation of general dental practitioners (GDPs) using a portfolio of evidence, held in a ring binder with six dividers, proforma sheets and plastic document pouches.

This second paper looks at the acceptability and ease of use of the portfolio format. The portfolio was acceptable, with caveats, to this small and self-selecting group.

'Softer' science was used to assess the acceptability of the portfolio model: 'an action research methodology', focus groups and semi-structured interviews, analysed by identifying main themes using an editing style. Some traditionalists may find themselves questioning the validity of this approach.

The assessors felt that the portfolio would have worked better in combination with appraisal, to assist the link from reflective practice to personal development plan. Research on appraisal for GDPs has already been carried out by some of this group.

Since these papers were submitted for publication, the GMC scheme has been withdrawn, following substantial criticism by the Shipman Enquiry, the Pillars of Clinical Governance have been modified, and the GDC's work has been put on hold awaiting the report by the Chief Medical Officer on Revalidation of the whole healthcare family.

This may cause a different approach to Revalidation to be adopted from that proposed by the authors, although they may be pleased to learn that it has many similarities to the model the GDC had been working towards, and since this scheme was tailored for Scottish GDPs, some minor alterations would be required to enable use of this format in the other home countries.

Revalidation is likely to be a contentious issue for GDPs, and more research such as this is required to assess its effectiveness, acceptability and value to both the profession and the general public.

J. Lafferty, General Dental Practitioner,

Sheffield (GDC Revalidation Working Group 2001-2005)

doi: 10.1038/sj.bdj.4813561 